\title{
A computational study of vortex-airfoil interaction using high-order finite difference methods
}

Magnus Svärd, Johan Lundberg and Jan Nordström

\section{Post Print}

N.B.: When citing this work, cite the original article.

Original Publication:

Magnus Svärd, Johan Lundberg and Jan Nordström, A computational study of vortex-airfoil interaction using high-order finite difference methods, 2010, Computers \&amp; Fluids, (39), 1267-1274.

http://dx.doi.org/10.1016/j.compfluid.2010.03.009

Copyright: Elsevier Science B.V., Amsterdam. http://www.elsevier.com/

Postprint available at: Linköping University Electronic Press http://urn.kb.se/resolve?urn=urn:nbn:se:liu:diva-68614 


\title{
A computational study of vortex-airfoil interaction using high-order finite difference methods
}

\author{
Magnus Svärd *, Johan Lundberg †and Jan Nordström ${ }^{\ddagger}$ \\ January 11, 2010
}

\begin{abstract}
Simulations of the interaction between a vortex and a NACA0012 airfoil are performed with a stable, high-order accurate (in space and time), multi-block finite-difference solver for the compressible NavierStokes Equations.

We begin by computing a benchmark test case to validate the code. Next, the flow with steady inflow conditions are computed on several different grids. The resolution of the boundary layer as well as the amount of the artificial dissipation is studied to establish the necessary resolution requirements. We propose an accuracy test based on the weak imposition of the boundary conditions that does not require a grid refinement.

Finally, we compute the vortex-airfoil interaction and calculate the lift and drag coefficients. It is shown that the viscous terms add the effect of detailed small scale structures to the lift and drag coefficients.
\end{abstract}

\section{Introduction}

Flows around multiple bodies are common in aerodynamic computations. Each body will disturb the free stream and the disturbances interact downstream of the body. To obtain good solutions for multi-body configurations,

${ }^{*}$ School of Mathematics, University of Edinburgh, King's Buildings, Mayfield Road, Edinburgh, EH9 3JZ, United Kingdom; e-mail: Magnus.Svard@ed.ac.uk

${ }^{\dagger}$ Department of Information Technology, Uppsala University, SE-751 05 Uppsala, Sweden; ABB AB, Nätverksgatan 3, Building 391, SE-721 59 Västerås, Sweden

${ }^{\ddagger}$ Department of Computational Physics, The Swedish Defense Research Agency, SE16490 Stockholm, Sweden; Department of Information Technology, Uppsala University, SE-751 05 Uppsala, Sweden; e-mail jan.nordstrom@foi.se 
the numerical scheme must accurately compute the flow around the bodies, as well as the wave propagation between the bodies. As a prototype of such problems, we will study a vortex-airfoil interaction. The vortex represents a structure generated by a body upstream of the airfoil.

In [3], the vortex-airfoil interaction problem governed by the Euler equations was studied. It was shown that high-order accuracy in space results in superior computational efficiency. However, while the Euler equations might be a good model for the vortex transport, it is less likely to be a good model in the interaction phase since viscosity plays an important role. The purpose of the present work is to study vortex-airfoil interaction governed by the Navier-Stokes equations and take viscous effects into account. Previously published results on vortex-airfoil interaction concern the inviscid case, [14].

The simulations have been carried out with a stable, high-order accurate (in both space and time), multi-block finite difference solver for the compressible Navier-Stokes and Euler equations, described in [9] and [11]. The finite difference scheme satisfies a Summation-by-Parts rule (SBP) and the Simultaneous Approximation Term (SAT) technique is used to weakly enforce the boundary conditions. The entire scheme for the Navier-Stokes equations can be proven linearly stable for the constant coefficient problem (see [9] and [11] and references therein). Linear stability of the constant coefficient problem leads to stability of the variable coefficient problem (see [5]) and stability of the linear variable coefficient problem leads to stability of non-linear problems with smooth solutions (see [8]). Hence, the proofs of stability in $[9,11]$ implies, via a mathematically stringent chain of arguments, stability of the problems we will study in this paper. This is corroborated in [10], where design order of accuracy (up to 5) was demonstrated for the present implementation.

In this article, we present a delicate validation test case to further demonstrate the accuracy of the code: the flow around a NACA0012 airfoil at Mach number $(M a) 0.5$ and Reynolds number $(R e) 5000$.

Next, we will increase the Reynolds number to 10000 and run with freestream inflow data (without a vortex). We will use that solution to quantify the accuracy of the present computations. This is done by studying the separation point, the resolution of the boundary layers and the effective amount of artificial diffusion.

Finally, we will compute the vortex-airfoil interaction at $M a=0.5$ and $R e=10000$. We monitor the lift and drag and show the differences from an inviscid computation. 


\section{The numerical method}

The numerical method (from now on referred to as SBP-SAT schemes) is described at length in [9] and [11]. Nevertheless, we will present its theoretical properties using the advection-diffusion equation and prove stability via an energy estimate. We will use two different boundary conditions; one mimicing a far-field boundary and the other one a wall.

We define the $L^{2}$-norm on $\left[x_{A}, x_{B}\right]$ as

$$
\|u\|_{2}^{2}=\int_{x_{A}}^{x_{B}} u^{2} d x .
$$

The $x$-axis is discretized with $N+1$ grid points such that $x_{i}=i h+x_{A}$, $i=0 . . N$ and $h=\left(x_{B}-x_{A}\right) / N$. A first-derivative approximation of a function $\mathbf{u}$ is written as

$$
P^{-1} Q \mathbf{u}=\mathbf{u}_{x}+T_{e},
$$

where $\mathbf{u}$ is the vector function

$$
\mathbf{u}=\left[u\left(x_{0}, t\right), u\left(x_{1}, t\right) \ldots u\left(x_{N}, t\right)\right]^{T}
$$

and $T_{e}$ is the truncation error. The derivative approximation, $P^{-1} Q \mathbf{u}$, is said to have the Summation-by-parts (SBP) property if:

1. $P$ is symmetric and positive definite. (In this article, we will only consider diagonal $P:$ s.)

2. $Q+Q^{T}=B$ where $B=\operatorname{diag}[-1,0 \ldots 0,1]$.

Second-derivatives will be approximated with the operator $D_{2}=P^{-1} Q P^{-1} Q$. We will also need the auxiliary vectors $\mathbf{e}_{\mathbf{N}}=[0 \ldots 0,1]^{T}$ and $\mathbf{e}_{\mathbf{0}}=[1,0, \ldots 0]^{T}$.

\subsection{Energy estimates}

Consider the one-dimensional advection-diffusion equation

$$
\begin{aligned}
u_{t}+a u_{x} & =\epsilon u_{x x}, \\
\alpha u\left(x_{A}, t\right)+\beta u_{x}\left(x_{A}, t\right) & =g^{A}(t), \\
u\left(x_{B}, t\right) & =0
\end{aligned}
$$

where the boundary condition at $x_{A}$ models an inflow or outflow boundary and the boundary condition at $x_{B}$ models a wall. We derive an energy 
estimate for (1) by multiplying by $u$ and integrating in space.

$$
\begin{aligned}
\frac{d}{d t}\|u\|^{2}+2 \epsilon\left\|u_{x}\right\|^{2} & =\left(a+\frac{2 \alpha}{\beta} \epsilon\right)\left(u\left(x_{A}, t\right)-\frac{\epsilon}{\beta\left(a+\frac{2 \alpha}{\beta} \epsilon\right)} g^{A}(t)\right)^{2} \\
& -\left(\frac{\epsilon}{\beta}\right)^{2}\left(\frac{1}{a+\frac{2 \alpha}{\beta} \epsilon}\right) g^{A}(t)^{2}
\end{aligned}
$$

An energy estimate is obtained if

$$
a+\frac{2 \alpha}{\beta} \epsilon<0
$$

We proceed to derive an energy estimate for the semi-discrete SBP-SAT scheme. Let $v$ denote the approximate solution, $v=\left[v_{0}(t), \ldots, v_{N}(t)\right]$. We discretize (1) as

$$
\begin{aligned}
v_{t}+a P^{-1} Q v-\epsilon D_{2} v & =\sigma^{A} P^{-1} \mathbf{e}_{\mathbf{0}}\left[\alpha v_{0}+\beta\left(P^{-1} Q v\right)_{0}-g^{A}\right] \\
& +\sigma^{B} P^{-1} \mathbf{e}_{\mathbf{N}}\left[v_{N}-0\right]
\end{aligned}
$$

where the terms on the right-hand side are the Simultaneous Approximation Terms (also referred to as SAT's or penalty terms) that will enforce the boundary conditions. The penalty parameters $\sigma^{A}$ and $\sigma^{B}$ will be determined with respect to stability. To simplify the analysis it will be useful to introduce $\sigma^{B}=\sigma_{V}^{B}+\sigma_{I}^{B}$. Furthermore, we define $\|v\|_{P}^{2}=v^{T} P v$. Multiply equation (3) by $v^{T} P$ from the left and add the transpose of the equation. We obtain

$$
\frac{d}{d t}\|v\|_{P}^{2}+2 \epsilon\left\|P^{-1} Q v\right\|_{P}^{2}=B T_{L}+B T_{I R}+B T_{V R}
$$

where the boundary terms are

$$
\begin{aligned}
B T_{L}= & a v_{0}^{2}-2 \epsilon v_{0}\left(P^{-1} Q v\right)_{0}+2 \sigma^{A} \alpha v_{0}^{2}+ \\
& 2 \sigma^{A} \beta v_{0}\left(P^{-1} Q v\right)_{0}-2 \sigma^{A} v_{0} g^{A}, \\
B T_{I R}= & -a v_{N}^{2}+2 \sigma_{I}^{B} v_{N}^{2} \\
B T_{V R}= & 2 \epsilon v_{N}\left(P^{-1} Q v\right)_{N}+2 \sigma_{V}^{B} v_{N}^{2} .
\end{aligned}
$$

For stability, we require that the right-hand side is bounded from above by data. Beginning with (6) we see that

$$
\sigma_{I}^{B} \leq \frac{a}{2}
$$


is a necessary condition. Next, we consider (5). The terms with $u_{0}\left(P^{-1} Q u\right)_{0}$ must cancel exactly since it is not possible to bound them in a quadratic form. Hence, we require that

$$
\sigma^{A}=\epsilon / \beta .
$$

For $g^{A} \neq 0$ and using (9), (5) results in condition (2).

The viscous part of the wall boundary penalty (7), can be expressed as a quadratic form

$$
\left.\mathrm{BT}_{V R}=-2\left(\begin{array}{ll}
v_{N} & \left(\mathrm{P}^{-1} \mathrm{Qv}\right.
\end{array}\right)_{\mathrm{N}}\right) \underbrace{\left(\begin{array}{cc}
-\sigma_{V}^{B} & -\frac{\epsilon}{2} \\
-\frac{\epsilon}{2} & 0
\end{array}\right)}_{A_{V}}\left(\begin{array}{c}
v_{N} \\
\left(\mathrm{P}^{-1} \mathrm{Qv}\right)_{\mathrm{N}}
\end{array}\right) .
$$

The expression (10) is negative if the matrix $A_{V}$ on the right-hand side is positive semi-definite. That can not be achieved in the present form. Therefore, we performed a mathematical manipulation (introduced in [1]) to obtain an energy estimate.

The idea is to borrow a quadratic term from $2 \epsilon\left\|P^{-1} Q u\right\|_{P}^{2}$ on the lefthand side of (4). Recalling that $P$ is diagonal with elements $P_{i i}=p_{i} h$, we can rewrite $\|v\|_{P}^{2}=\sum_{i=0}^{N} v_{i}^{2} p_{i} h=\sum_{i=0}^{N-1} v_{i}^{2} p_{i} h+p_{N} v_{N}^{2} h=\|v\|_{\tilde{P}}^{2}+p_{N} v_{N}^{2} h$. Carrying out these changes, the term $2 \epsilon\left\|P^{-1} Q v\right\|_{P}^{2}$ in (4) turns into $2 \epsilon\left\|P^{-1} Q v\right\|_{\tilde{P}}^{2}$ and (10) becomes

$$
\begin{aligned}
\tilde{\mathrm{BT}}_{V R} & =\mathrm{BT}_{V R}-2 \epsilon h p_{N}\left(P^{-1} Q v\right)_{N}^{2} \\
& =-2\left(\begin{array}{ll}
v_{N} & \left(\mathrm{P}^{-1} \mathrm{Qv}\right)_{\mathrm{N}}
\end{array}\right) \underbrace{\left(\begin{array}{cc}
-\sigma_{V}^{B} & -\frac{\epsilon}{2} \\
\frac{\epsilon}{2} & \epsilon h p_{N}
\end{array}\right)}_{\tilde{A}_{V}}\left(\begin{array}{c}
v_{N} \\
\left(\mathrm{P}^{-1} \mathrm{Qv}\right)_{\mathrm{N}}
\end{array}\right) .
\end{aligned}
$$

$\tilde{A}_{V}$ is positive definite if

$$
\sigma_{V}^{B}<-\frac{\epsilon}{4 h p_{N}} .
$$

The above derivation can be summarized in the following proposition.

Proposition 2.1 The scheme (3) is stable if (2), (8), (9) and (12) hold.

Without going into detail, we conclude that when $\epsilon \rightarrow 0$, some terms of the penalty parameters will vanish, leaving only the conditions that enforce the well-posed boundary conditions for the inviscid equation. We also point to the fact that the effect of the $\epsilon$ dependent terms of the penalty parameters will diminish as $h$ grows, suggesting that the boundary conditions behave as their inviscid counterparts on coarse meshes. 
Details of the above argument, generalized to the Navier-Stokes equations, can be found in [9] and [11]. For the Navier-Stokes equations computed on a fixed grid, the wall boundary condition will approach a slip condition as $R e \rightarrow \infty$. Moreover, if the grid is coarsened and $R e$ is kept fixed, the solution will behave more like a slip condition.

\subsection{Artificial dissipation}

The following form of artificial dissipation was derived in [4],

$$
A_{2 p}=(-1)^{p+1} \tilde{P}^{-1} \tilde{D}_{p}^{T} B_{p} \tilde{D}_{p}
$$

where $\tilde{P}=\Delta x^{-1} P$ and $\tilde{D}_{p}=h^{p} D_{p}$ ( $D_{p}$ is a $p$ th-order derivative). The (symmetric part of the) matrix $B_{p}$ is required to be positive semi-definite.

We choose $\left(B_{p}\right)_{i i}=\left|\bar{v}_{i}\right|+c_{i}$, where $c_{i}$ is the local speed of sound and $\left|\bar{v}_{i}\right|$ is the magnitude of the local velocity vector. The artificial dissipation, $A_{2 p} v$, is added to a $2 p$ th-order accurate discretization of the Navier-Stokes equations. This choice will not increase the width of the stencil and it preserves the overall order of accuracy.

Furthermore, it is trivial to show that the proposed form results in a damping term in the energy estimate and hence it conforms precisely with the SBP theory. (We refer to [4] for more details.)

\subsection{Grid generation and parallelization}

Various grids around the NACA0012 airfoil will be used. They all have the same principal structure and consist of 12 blocks (see Figure 1). Each block is a smooth grid. The only requirement is that the grid lines match along each interface, but they need not be smooth across the interface. (See [7] for a stability proof.) 


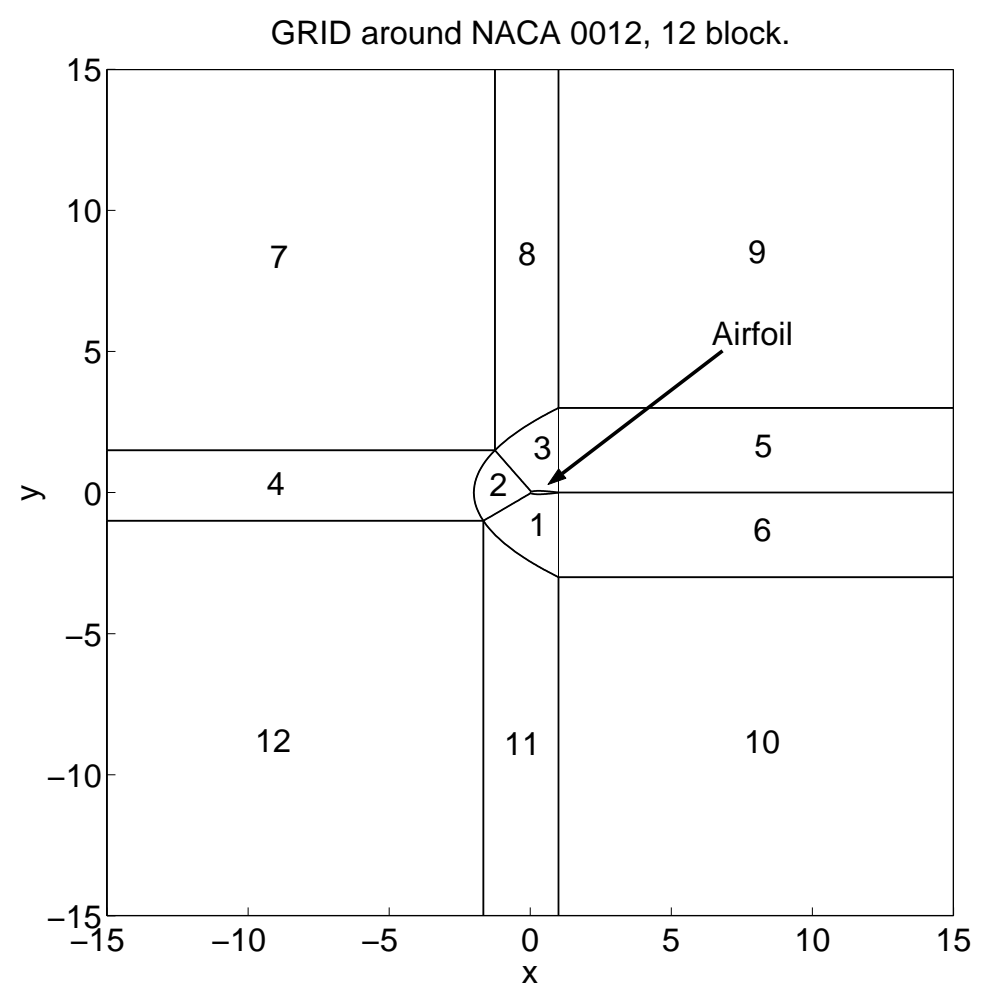

(a) The entire domain

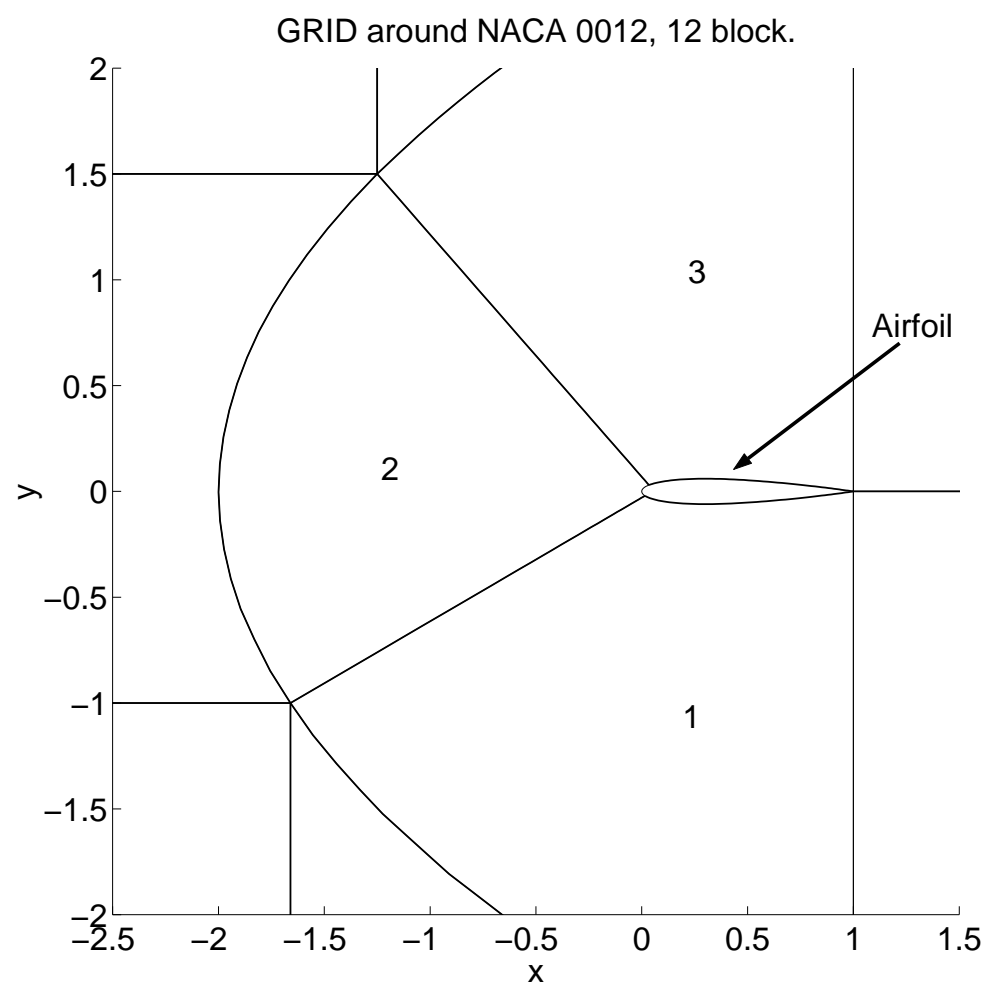

(b) Zoom of the airfoil 7

Figure 1: The multi-block computional grid. 


\begin{tabular}{|c|c|c|}
\hline Name & $\Delta_{\min }$ & Nodes on airfoil \\
\hline Reference & $5 \mathrm{e}-5$ & 350 \\
\hline Grid A & $1 \mathrm{e}-4$ & 350 \\
\hline Grid A (coarse) & $2 \mathrm{e}-4$ & 175 \\
\hline Grid B & $4 \mathrm{e}-4$ & 350 \\
\hline Grid C & $16 \mathrm{e}-4$ & 350 \\
\hline Grid D & $64 \mathrm{e}-4$ & 350 \\
\hline
\end{tabular}

Table 1: Computational grids. The normal resolution (and hence the number of grid points) changes in the grid blocks surrounding the airfoil.

To study the resolution requirements close to the airfoil, we use several different grids in the blocks 1,2,3 in Figure 1. We will only change the resolution in the normal direction and keep the number of grid points around the airfoil constant. Moreover, the resolution near the outer boundary of blocks $1,2,3$ is also kept constant such that the resolution only changes in the boundary layer. Finally, the resolution in the $y$-direction in blocks 5 and 6 will change as a consequence of the changes in 1,2 and 3 due to the requirement that grid lines match across an interface. The different grids used in this article are listed in Table 1.

To obtain solutions in a reasonable amount of time, the 12-block grid was split and run on 28 processors (14 AMD Opteron nodes). We stress that the subdivision is purely artificial and does not change the numerical results.

The splitting was done to achieve a good load balance on Grid A. On all other grids we use the same splitting, which results in a non-optimal load balance since Grids B,C,D contain fewer points in some of the blocks.

\section{Computations}

Throughout this section, we will use two different SBP-SAT schemes. The first is a scheme with internal fourth-order accuracy in space. The truncation errors near the boundaries are of order two for the inviscid terms and one for the viscous terms. As a consequence, the global order of accuracy is three and we will refer to this scheme as the 3rd-order scheme. The other scheme has internal order of eight with truncation errors of order four (inviscid) and three (viscous) near the boundaries, and the global order is five in space. It will be termed the 5th-order scheme. (For a derivation of global order of accuracy see [10].) We remark that the global orders of accuracy (5 and 3 , in our cases) are the convergence rates that can be observed pointwise everywhere in the domain, including the boundaries. 
Furthermore, all computations have been carried with a 4th-order accurate low-storage explicit Runge-Kutta scheme for the temporal discretization.

Remark There is a globally second-order accurate version of the SBP-SAT scheme available that is the standard central finite-difference scheme with a specific boundary closure. However, we choose to not include computations with that scheme since second-order of accuracy is not sufficiently accurate for vortex propagation, see [3].

\subsection{Validation test case}

It was shown in [10] that the present technique indeed converges with the correct convergence rates for the different orders of accuracy. Moreover, the code has been validated in [11] for the flow around a cylinder and good agreement with previously published data was reported.

To further demonstrate the strength of this technique, we have included the following benchmark case: flow around the NACA0012 airfoil at $R e=$ $5000, M a=0.5$ and 0 degrees angle of attack. We compute this flow using the 3rd-order scheme on Grid A (fine) and on every second point of Grid A (coarse). At the far-field boundaries we enforce the free-stream data using the characteristic boundary conditions derived in [9]. On the airfoil, no-slip boundary conditions proposed in [11] are applied.

This flow case has previously been studied in $[12,13,15,6]$ and we will refer to it as the Swanson and Turkel (or S\&T) test case. For this test case, the flow separates but no wake instabilities should appear. Excessive artificial diffusion increases the effective Reynolds number and moves the separation point downstream. Furthermore, the test case is sensitive to asymmetries in the solution procedure, which easily can trigger vortices and a steady state would not be obtained.

We define the separation point as the first grid point at which the flow turns backwards. The separation points computed with our scheme are shown in Table 2 along with previously published data. Our results agree to within one grid point of the reference data, which is the best we can achieve. In Figure 2, we observe that no wake instabilities occur.

Remark Our way of defining the separation point is conservative and leaves no ambiguity as to how it is obtained. Of course, one may use different interpolation techniques to estimate the separation point between grid points, but we prefer this stricter method. 


\begin{tabular}{|r|r|r|r|r|}
\hline Grid & Nodes on airfoil & $\Delta_{y, \min }$ & Separation point & $\Delta_{x}$ \\
\hline Coarse & 175 & $2 \mathrm{e}-4$ & 0.828 & 0.018 \\
\hline Fine & 350 & $1 \mathrm{e}-4$ & 0.819 & 0.009 \\
\hline S\&T 85 & 128 & $3 \mathrm{e}-4,6 \mathrm{e}-4$ & 0.817 & - \\
S\&T 87 & 129 & $6 \mathrm{e}-4$ & 0.811 & - \\
Venkatakrishnan & - & - & $0.81-0.824$ & - \\
Mittal & - & - & 0.813 & - \\
\hline
\end{tabular}

Table 2: Results for the validation test (NACA0012, $M a=0.5, R e=5000$ ) compared with [12] (S\&T85), [13] (S\&T87), [15] (Venkatakrishnan) and [6] (Mittal). $\Delta_{y, \min }$ is the minimum grid size in the normal direction of the airfoil. $\Delta_{x}$ is the grid size along the airoifl. The fine grid is Grid A and the coarse is every second point of Grid A. Our results are within one grid cell of those of the references.

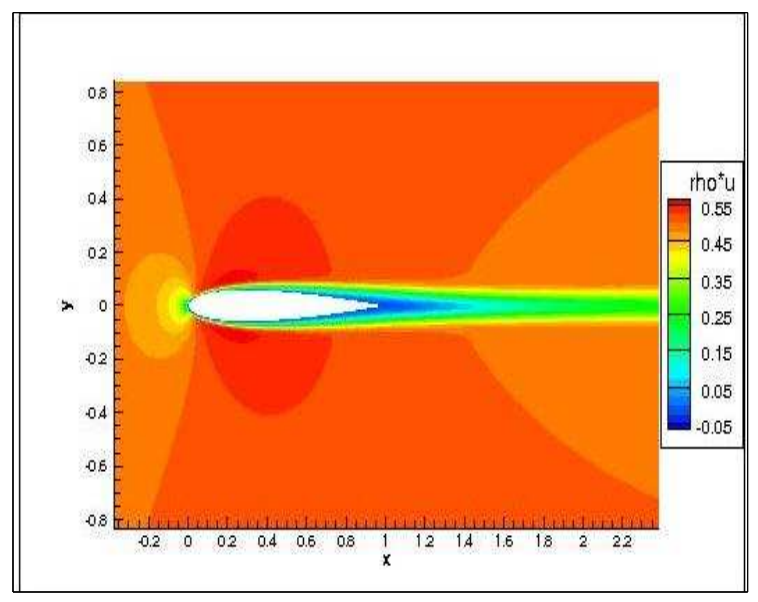

(a) Velocity in the x-direction

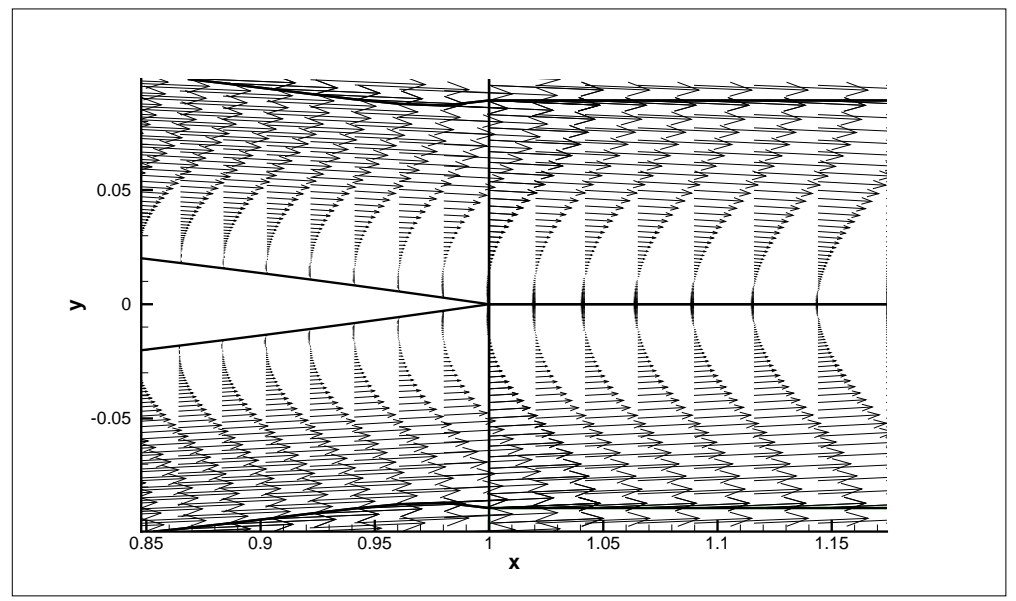

(b) Separation and back flow

Figure 2: The validation test case (16ACA0012 at $R e=5000, M a=0.5$ ) computed with a 3rd-order scheme. 
In Figure 3 a zoom of the separation region can be seen. In both the coarse and the fine grid the separation occurs in the cell immediately following $x=0.81$. We also point to the fact that in all plots the velocity vector at the boundary is too short to be seen, despite the substantial zoom level. (Compare with the reference vector of length $1 e-5$.) The no-slip condition is almost exactly fulfilled even with the weak enforcement of the boundary condition. We emphasize that with this methodology, no optimization of any parameters is needed. The test was setup and computed once. We conclude that this is a very powerful feature and strongly supports that our code accurately computes approximations to the correct solution also in cases where the solution is not known.

\subsection{Steady inflow data}

Next, we consider the case with steady inflow data at $M a=0.5$ and $R e=$ 10000. Again, we employ the 3rd-order scheme. Solutions were computed for all grids in Table 1 . The computations were initialized with constant freestream values in the entire domain. Tail vortices and back flow appeared in the numerical solution after some time as is seen in Figure 4 for Grid A. (The solutions on the other grids behave similarly.) The computations were run until a constant frequency vortex shedding was achieved at about 90 time units. 

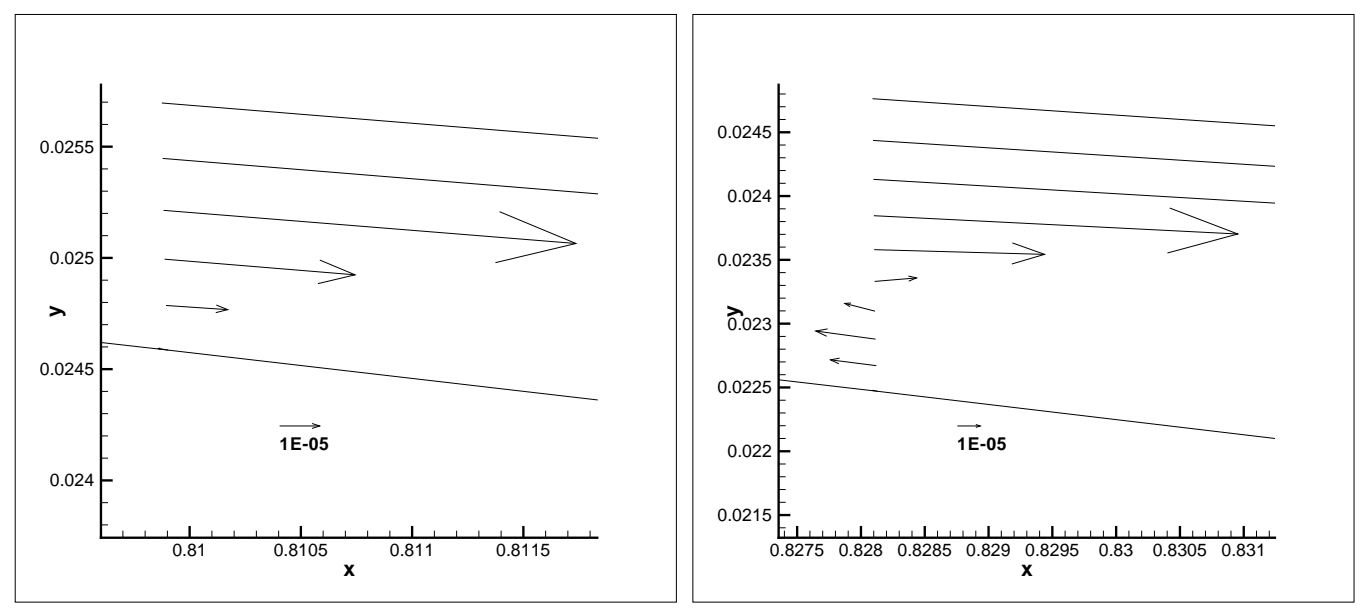

(a) The point before separation. Coarse (b) The point after separation. Coarse grid. grid.
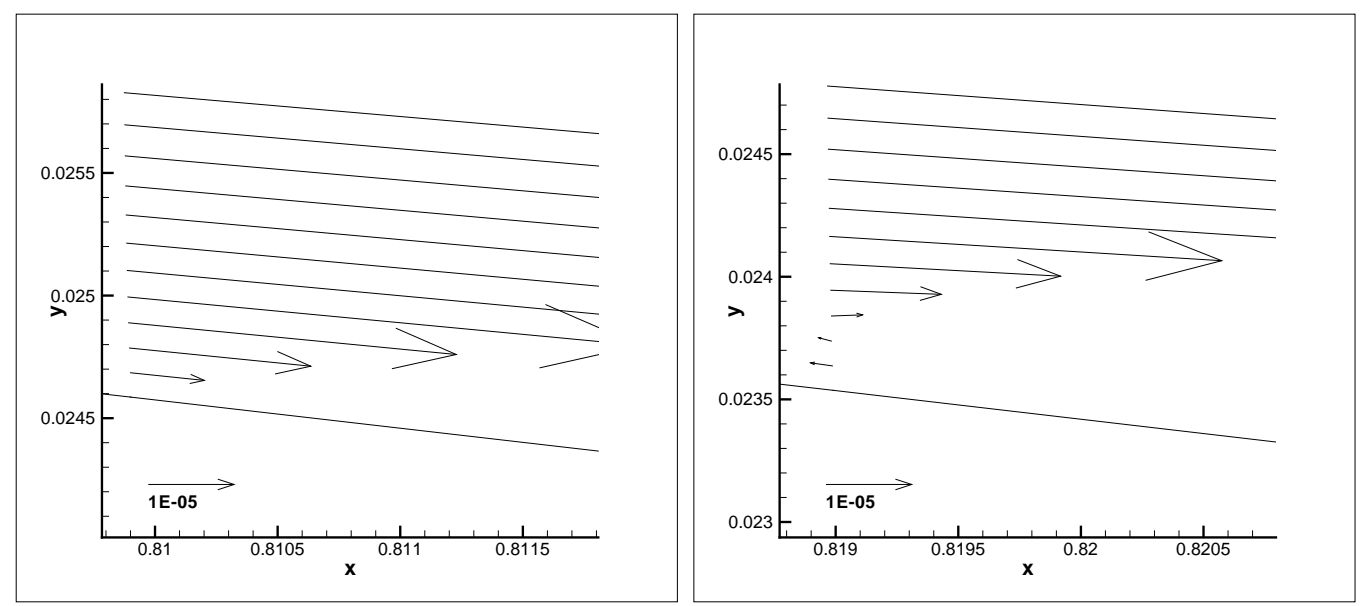

(c) [The point before separation. Fine grid. (d) [The point after separation. Fine grid.

Figure 3: The validation test case (NACA0012 at $R e=5000, M a=0.5$ ) computed with a 3rd-order scheme. Zoom of velocity vectors at the separation region (including a reference vector). 


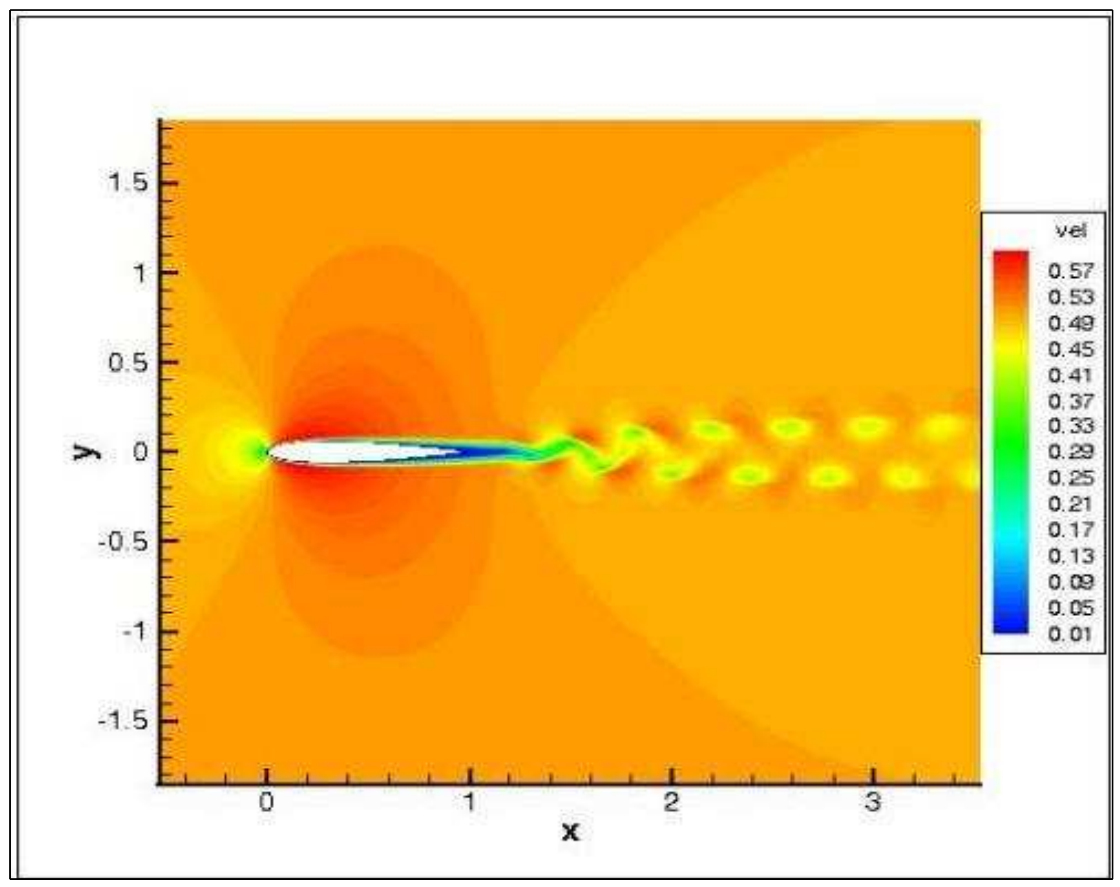

(a) Tail vortices

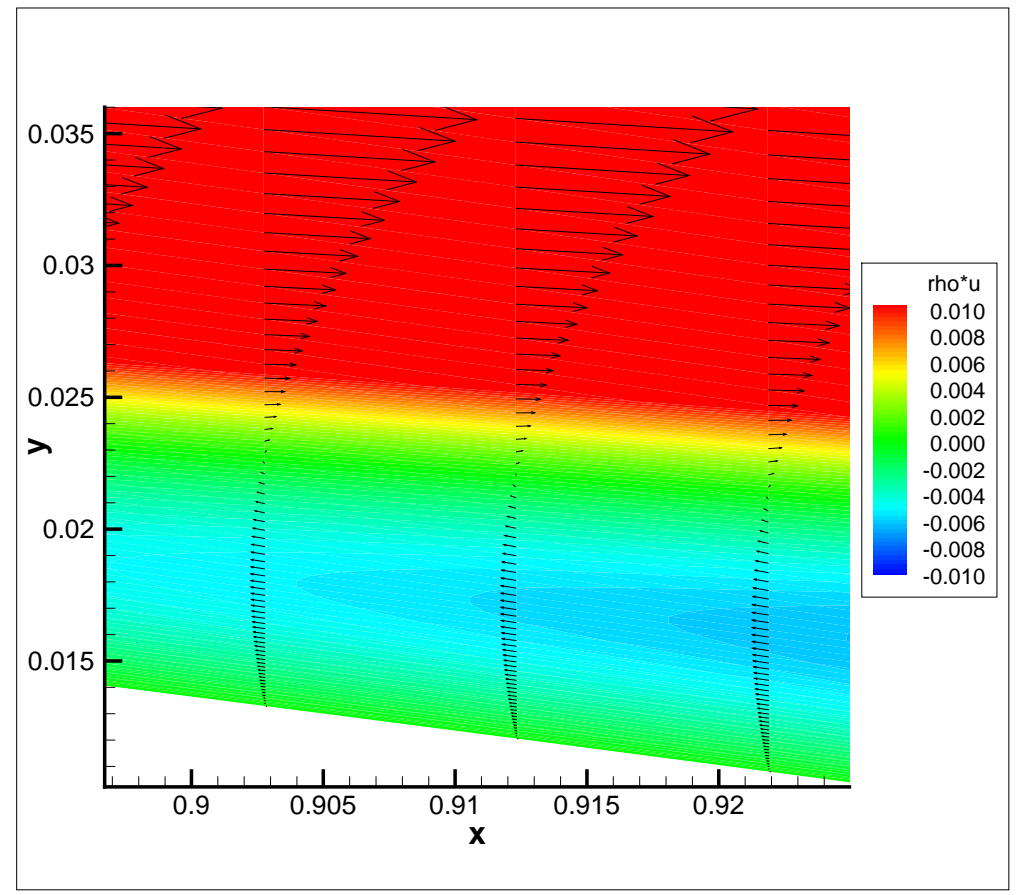

(b) Separation region

Figure 4: Solution at $t=90$ for Grid A computed with a 3rd-order scheme. Figure b is a close up of the separation region at the airfoil. 
The reference grid, Grid A and B all have similar boundary layer profiles and the no-slip condition is enforced along the airfoil. It is tempting to assume that the boundary layers are well resolved on those grids. (See Figures 5,6 and 7,8 .) We also observe that for the two coarser grids (C and D), the wall boundary condition behaves more like a slip condition, i.e., they are likely to be less resolved. Below we will show that the error of the noslip condition, indeed is a way to quantify the accuracy of a given solution computed with this technique.

In Table 3 the points of separation are listed for the different grids. For Grid A and B the separation points are the same as for the reference grid and then it moves slightly towards the tail for the coarser grids (C and D). This measure of accuracy suggests that for the 3rd-order scheme, Grids A and $\mathrm{B}$ are resolved, while Grid $\mathrm{C}$ is on the verge of being resolved. This is consistent with the observation in the Figures 5,6 and 7, 8 regarding the enforcement of the no-slip condition.

Remark We emphasize that the weak imposition is a very robust technique. Despite the boundary layer being under-resolved, the scheme produces a reasonably accurate solution on both Grid C and D. This was reported in [11], where it also was shown that more standard techniques often fail in such cases and become unstable. 


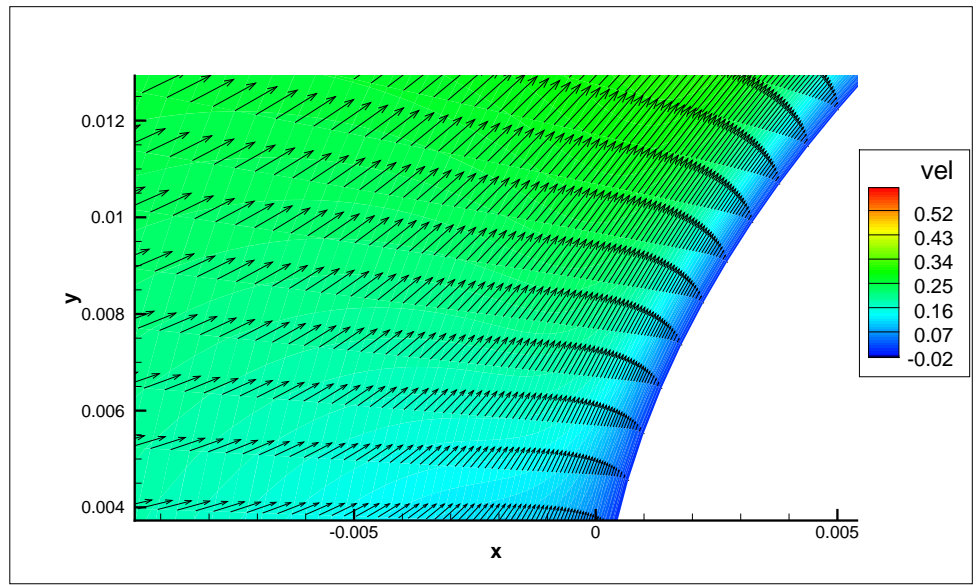

(a) Grid A

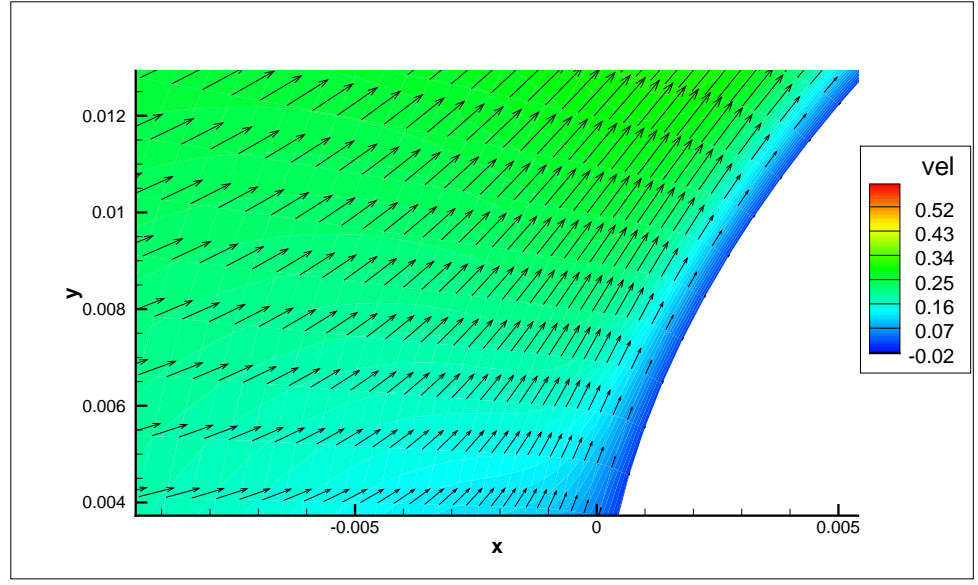

(b) Grid B

Figure 5: Solutions computed with a 3rd-order scheme on different grids. The figures show a region close to the nose of the airfoil. 


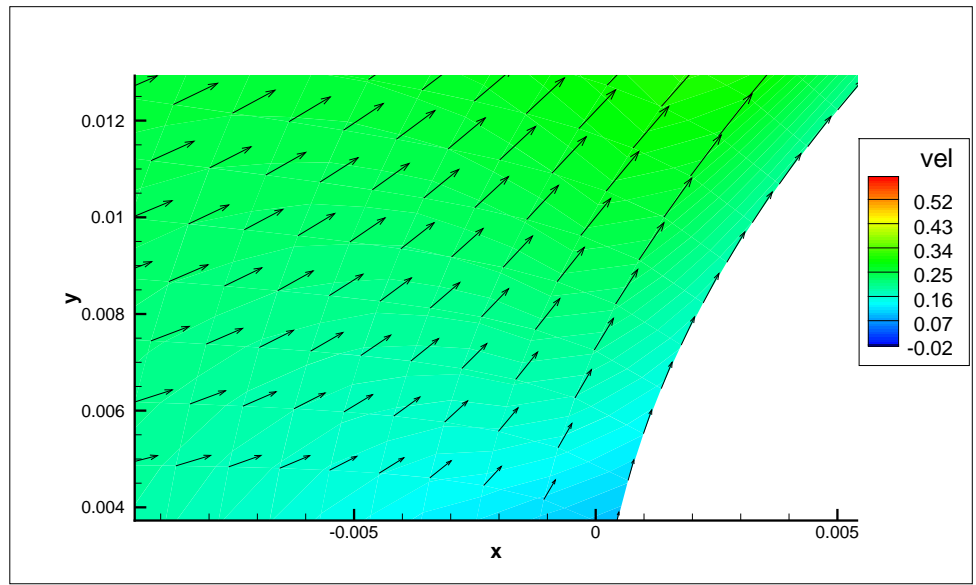

(a) Grid C

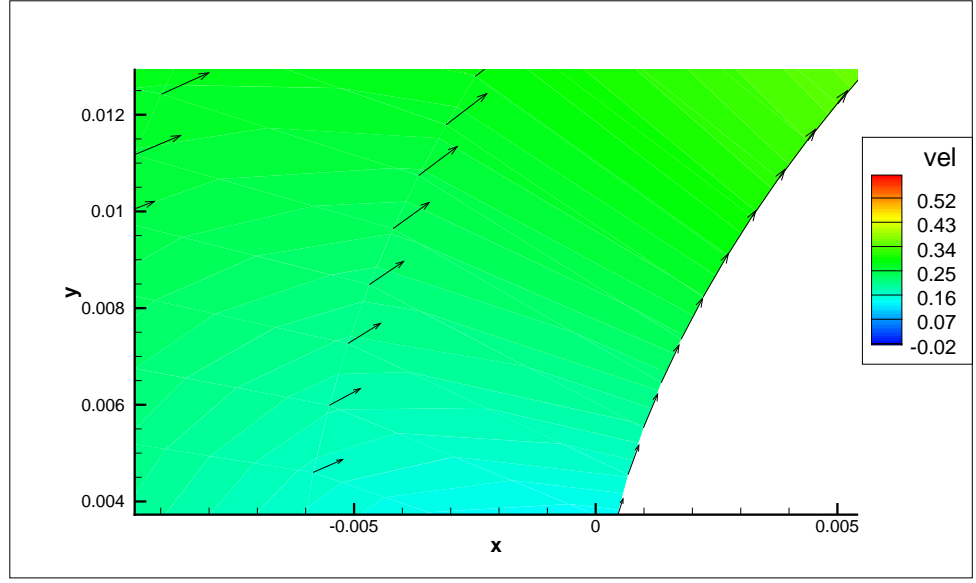

(b) Grid D

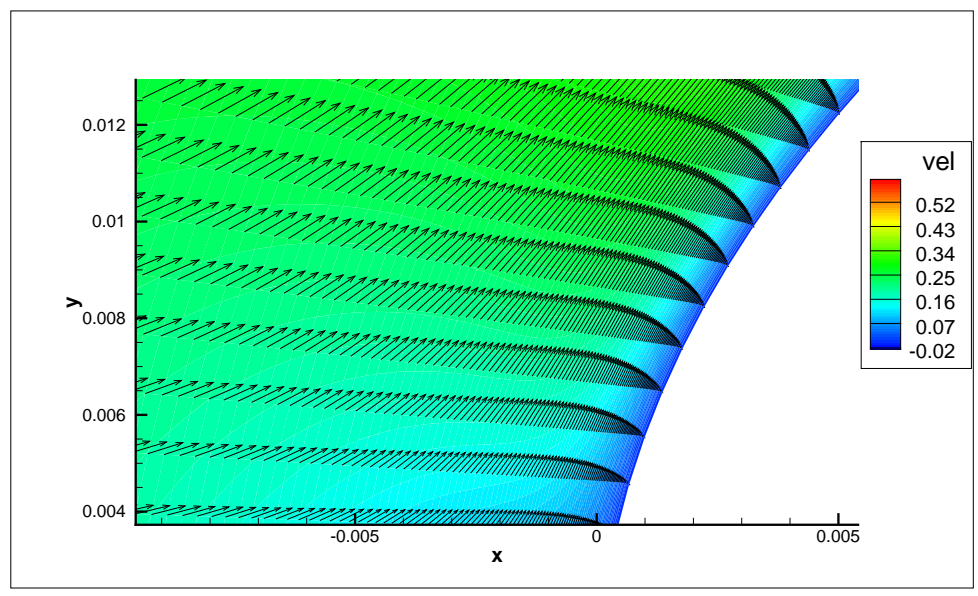

(c) Reference grid

Figure 6: Solutions computed with a 3rd-order scheme on different grids. The figures show a region close to the nose of the airfoil. 


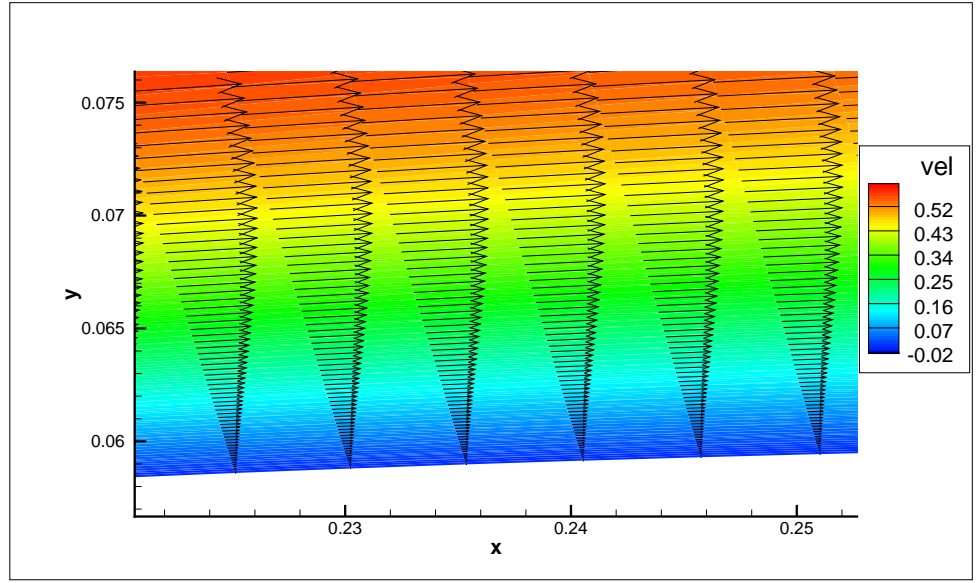

(a) Grid A

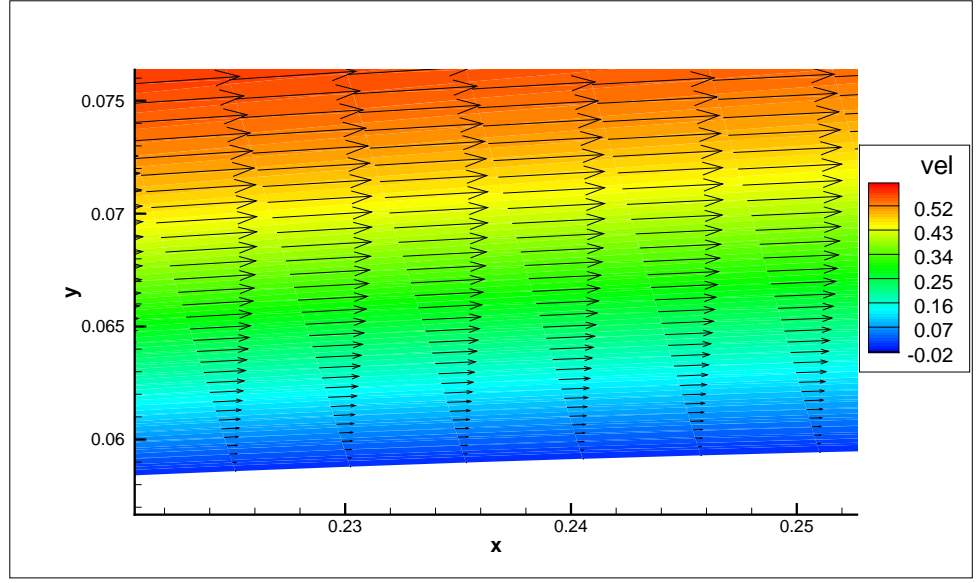

(b) Grid B

Figure 7: Solutions computed with a 3rd-order scheme on different grids. The figures show a region in the middle of the airfoil 


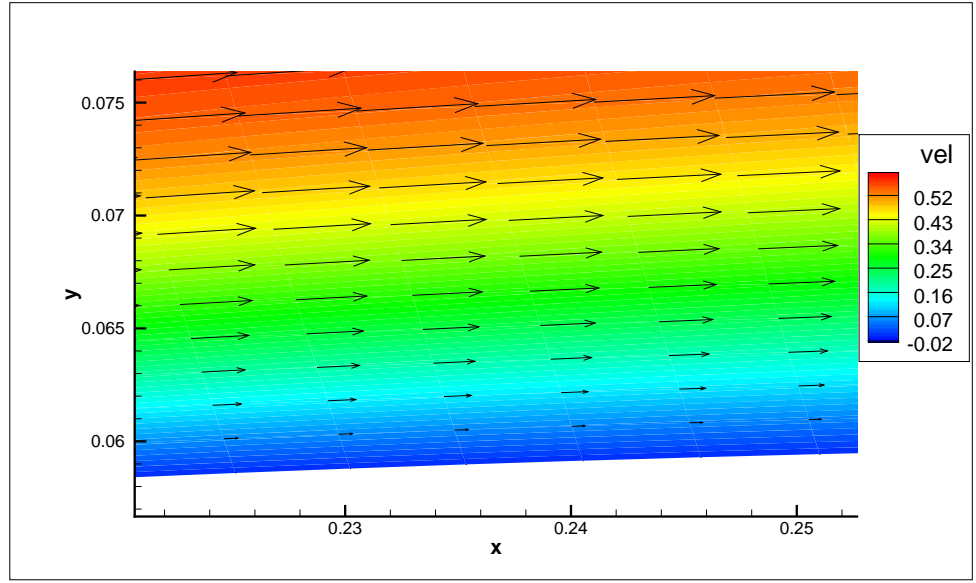

(a) Grid C

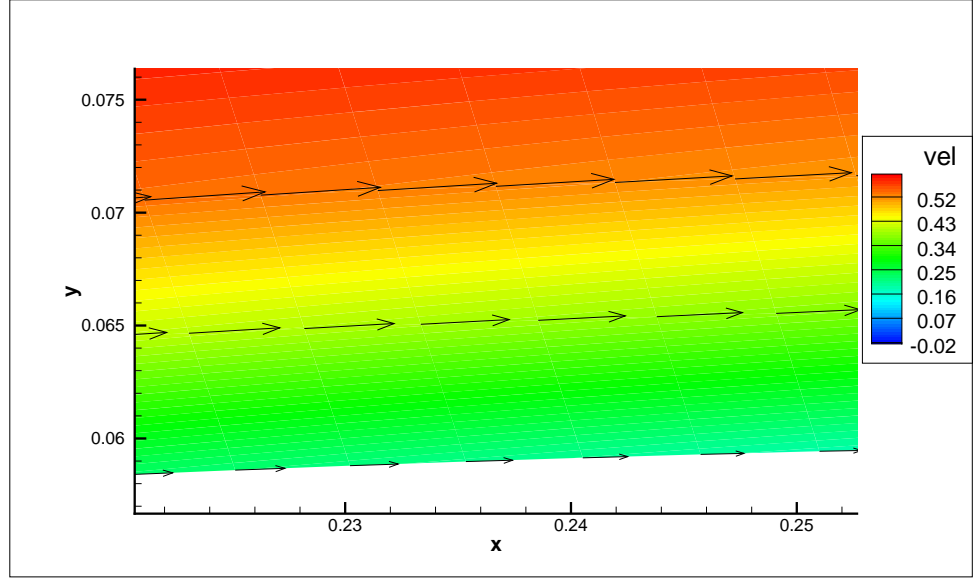

(b) Grid D

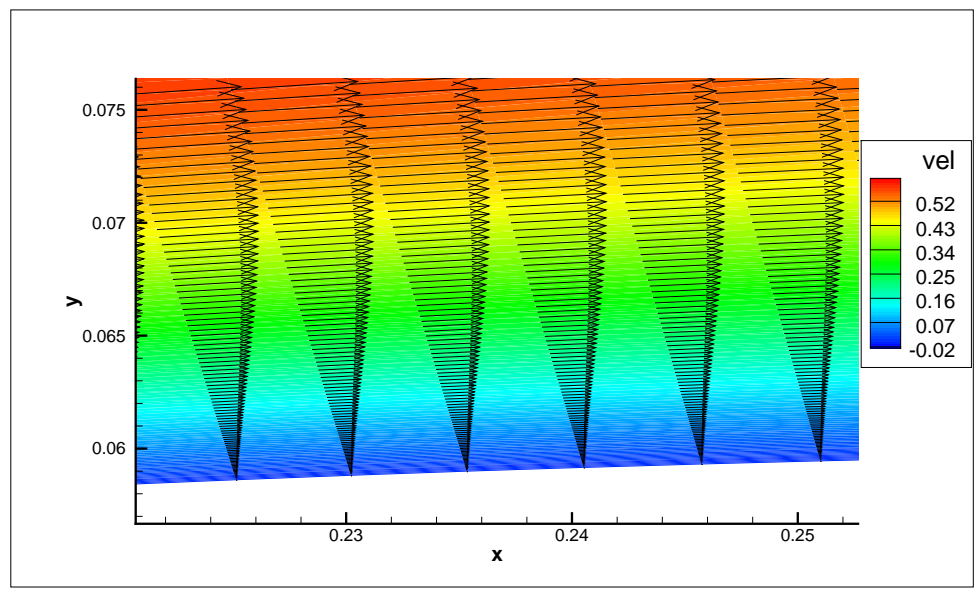

(c) Reference grid

Figure 8: Solutions computed with a 3rd-order scheme on different grids. The figures show a region in the middle of the airfoil 


\begin{tabular}{|c|c|c|c|c|c|}
\hline Grid & Reference & A & B & C & D \\
\hline Point of separation & 0.739 & 0.739 & 0.739 & 0.756 & 0.747 \\
\hline
\end{tabular}

Table 3: Point of separation with steady inflow condition computed with the 3rd-order scheme on different grids.

In [11] it was shown that the 5th-order scheme was the most efficient scheme (in terms of computational time and accuracy) to compute the shedding behind a circular cylinder. To study the impact on the solution by order of accuracy, we have run the 5th-order scheme on Grid C. Without showing the results, we just state that the separation point is the same as for the 3rd-order scheme on Grid C. This is also reflected in the boundary layers, which are only slightly better resolved than for the 3rd-order scheme.

As a final measure of accuracy, we compare the ratio, artifical diffusion/total diffusion. If the ratio is small, the physical viscosity dominates, which indicates that the accuracy is good. If the artificial diffusion dominates it is a sign of under-resolution. We calculated the ratio at 4 different points in the boundary layer on the airfoil and at 1 point in the wake behind the airfoil. The results support the previous conclusion. The 3rd-order scheme on Grid A has a very low contribution from the artificial viscosity and the solution is determined by the physical terms. (The range of the ratio is: 0.000-0.072) The same scheme in Grid $\mathrm{C}$ shows the artificial diffusion has a substantial impact. (0.028-0.965) The 5th-order scheme on Grid C results in the ratios, 0.017-0.955, which again is consistent with the previous conclusion that the 5th-order scheme is about as accurate as the 3rd-order on Grid $\mathrm{C}$ for this particular problem. (Still this does not necessarily rule out the 5th-order scheme, since it might be more efficient when time dependent structures, such as the vortex, appear in the solution.)

Returning to our inital assumption that the resolution of the no-slip condition is an indicator of the overall accuracy, we conclude that all the above accuracy tests support that. By looking at the boundary layers and the extent to which the no-slip condition is satisfied, we drew the conclusion that the solutions on Grid A and B were resolved, Grid D under-resolved and Grid $\mathrm{C}$ somewhere in between. The separation points and the sizes of the artificial diffusion also pointed at the same conclusion. The impact of this is significant. Computing the solution on a series of finer grids is a standard way of assessing the quality of a solution. But for practical computations that is most often not feasible due to the computational costs. What is desirable is a way to measure the accuracy of a given computation. With the present technique of imposing the boundary conditions weakly, we have such a measurement at hand. The above observations shows a strong correla- 
tion between the resolution of the boundary layers and the no-slip condition (which can be evaluated for a single computation), and the accuracy of the solution.

Remark In [11], the flow around a circular cylinder was computed with the same numerical schemes. The weak enforcement was studied and convergence at no-slip boundaries was shown. The results in [11] supports the conclusions in the present article.

\subsection{Vortex-airfoil interaction}

To study the vortex-airfoil interaction, we will use an exact vortex solution to the Euler equations in free space. The full expressions for the Euler vortex are found in [2]. To indicate its properties we show the tangential velocity

$$
v_{\theta}=\frac{\epsilon r}{2 \pi} \exp \left(\frac{1-r^{2}}{2}\right) .
$$

We will use the radius $r=0.5$ and strength $\epsilon=0.5$. The solution is used as inflow data with the vortex center starting 3 length units outside the left boundary $($ at $(x, y)=(-18,0))$. The vortex propagates with the flow $M a=0.5$ towards the airfoil at $x=0$. We compute the solution using the 3rd-order scheme.

In Table 4, we show the movement of the separation point. The unaffected separation point is 0.739 (see Table 3 ), which is the value before and after the vortex passage. Note also that $t=0$ refers to the time the center of the vortex enters the domain and in a free stream the vortex will be at the nose at about $t=30$, but the effect on the airfoil is seen earlier.

Next, we study the forces on the airfoil by integrating the pressure, $p$, and viscous stress, $\overline{\bar{\tau}}$, over the surface of the airfoil, $\partial W$,

$$
\mathbf{F}=\int_{\partial W}(\overline{\bar{\tau}} \mathbf{n}-p \mathbf{n}) d s .
$$

The lift and drag forces are the $x$ and $y$ components of the total force. ( $x$ is the flow direction.)

$$
\mathbf{F}=F_{D} \hat{\mathbf{x}}+F_{L} \hat{\mathbf{y}}
$$

The lift and drag coefficients are defined as

$$
C_{L}=\frac{F_{L}}{\frac{1}{2} \rho_{\infty} u_{\infty}^{2} l_{C}}, \quad C_{D}=\frac{F_{D}}{\frac{1}{2} \rho_{\infty} u_{\infty}^{2} l_{C}},
$$




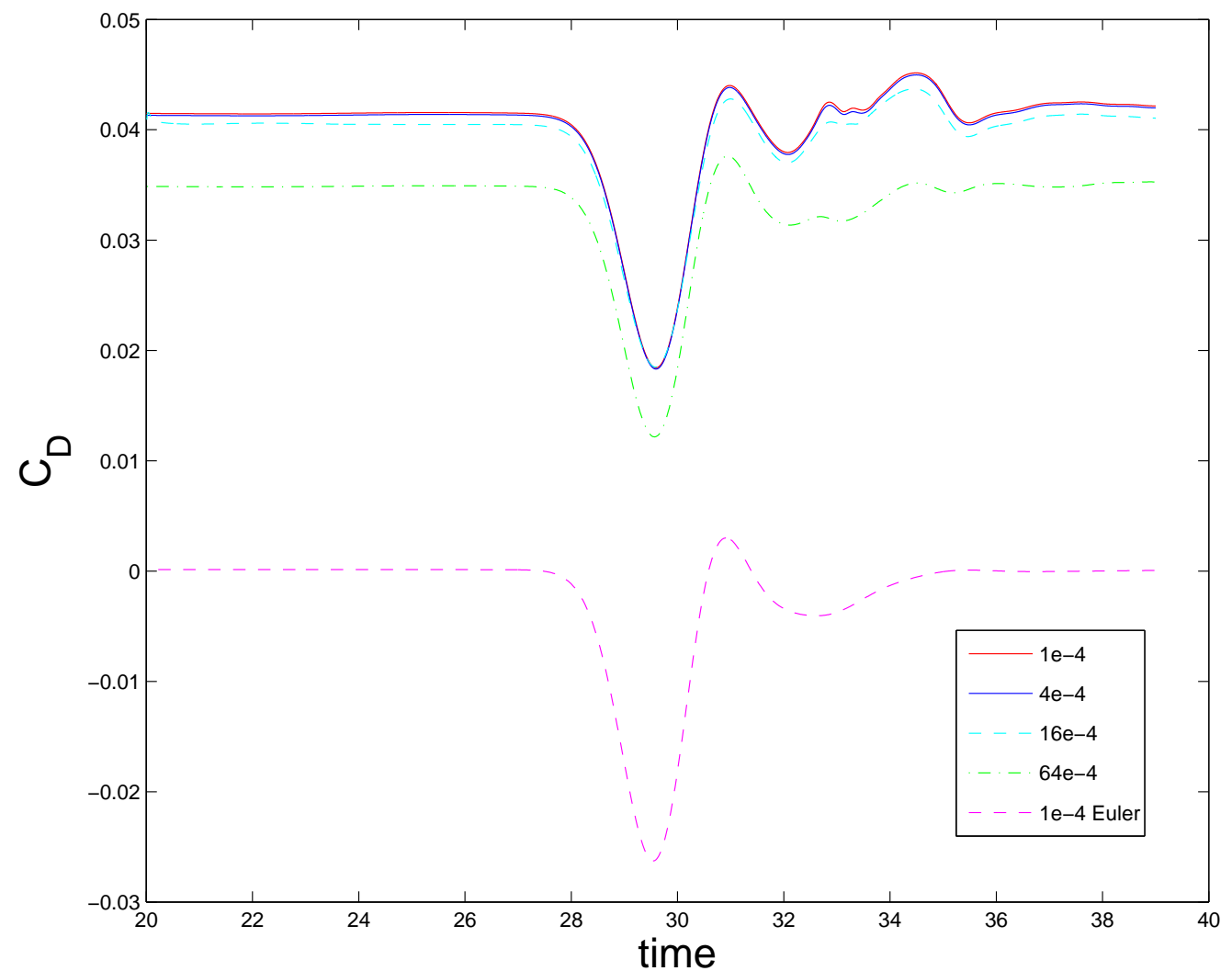

Figure 9: The drag coefficient of solutions computed with the 3rd-order scheme on Grids A-D. As a reference we show the Euler solution on Grid A. 


\begin{tabular}{|l|l|l|}
\hline Time & $\begin{array}{l}\text { Point of separa- } \\
\text { tion on upper side }\end{array}$ & $\begin{array}{l}\text { Point of separa- } \\
\text { tion on lower side }\end{array}$ \\
\hline 26 & 0.756 & 0.745 \\
\hline 27 & 0.756 & 0.745 \\
\hline 28 & 0.765 & 0.718 \\
\hline 29 & 1.000 & 0.641 \\
\hline 30 & No separation & 0.419 \\
\hline 31 & 0.922 & 0.490 \\
\hline 32 & 0.756 & 0.763 \\
\hline 33 & 0.515 & 0.818 \\
\hline
\end{tabular}

Table 4: Point of separation during the vortex passage computed with the 3rd-order scheme on Grid B. The point of separation before and after the vortex is at 0.739

where $\rho_{\infty}$ and $u_{\infty}$ are the free stream density and velocity, and $l_{C}$ is the chord length. The drag coefficient is shown in Figure 9. The drag coefficient curves for the two finest grids (A and B) are almost indistinguishable, implying that the solutions are well-resolved. We would like to draw attention to the appearance of small scale variations for the fine grids, which diminish as the grids coarsen and are absent in the Euler computation. Furthermore, we note that the drag coefficient for the under-resolved grids quickly decreases towards 0 (away from the interaction phase). However, Grid D still has a substantial free-stream drag since the boundary layers are not under-resolved everywhere. Nevertheless, this is another indication that the wall boundary conditions behaves increasingly like a slip condition as the grid is coarsened.

The lift coefficient is plotted in Figure 10 and shows that the three finest grids (A,B and C) seem to resolve the interaction well. The lift coefficient on Grid D lacks a lot of finer structures implying that some of the dynamics induced by the viscosity is not captured on this grid. The lack of finer structures is even more pronounced in the Euler solution, which is clearly different.

Remark In the above study we have excluded the reference grid, since the simulation time was too long and the results from steady inflow indicated that Grid A is sufficiently fine. This is also confirmed by the fact that the solutions on Grid A and B are very similar. 


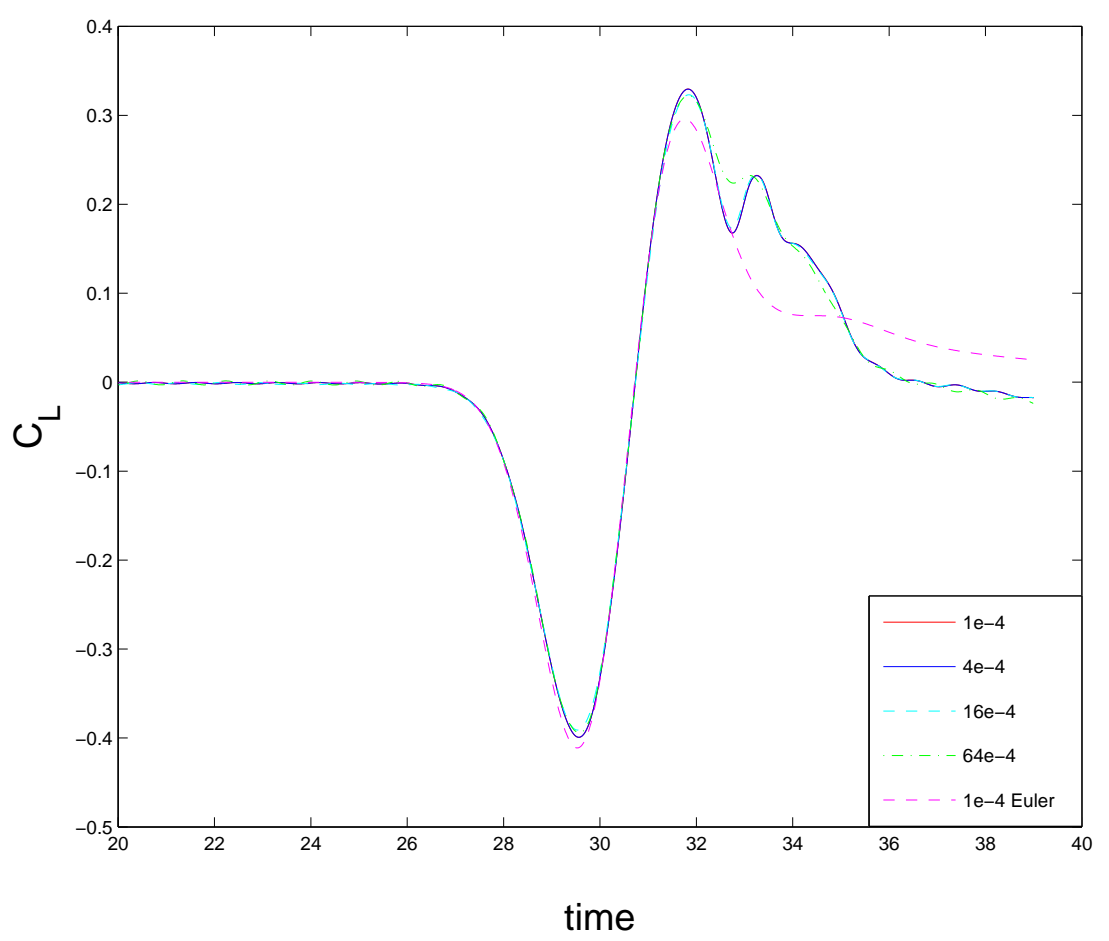

(a) Lift coefficients

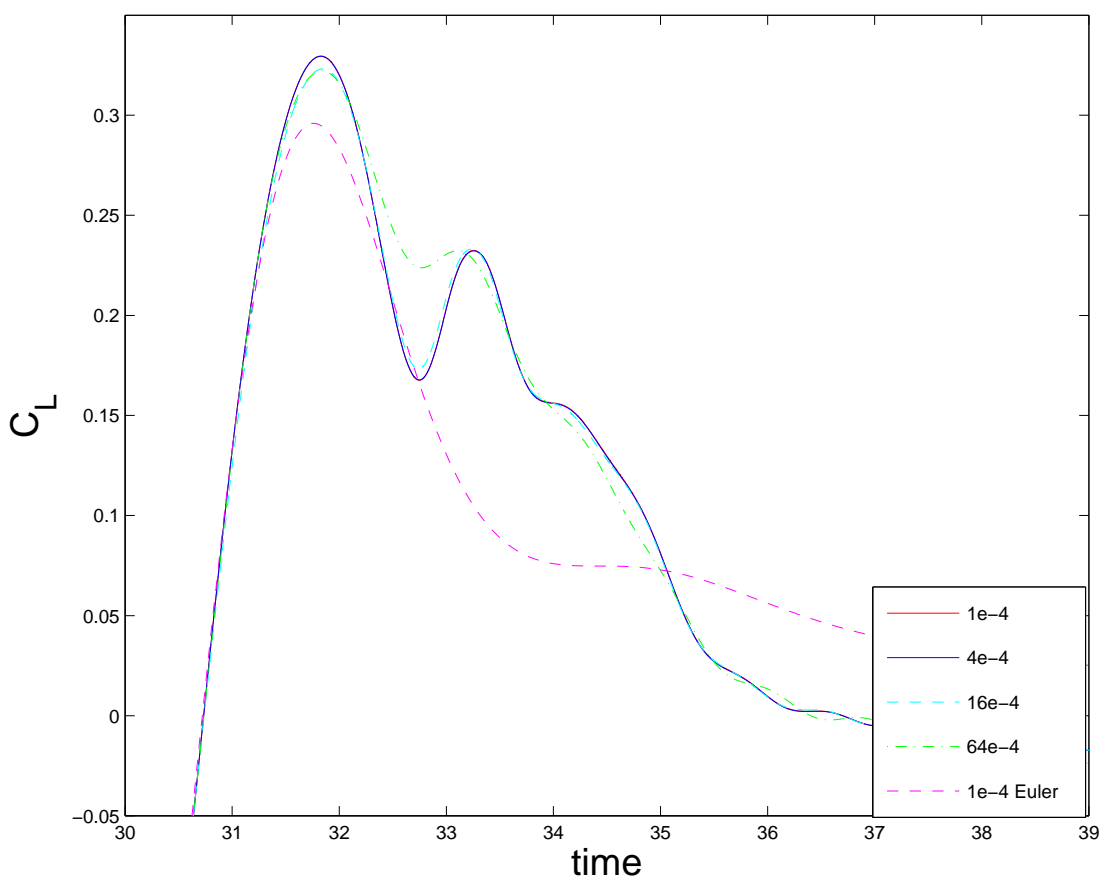

(b) A zoom on the lift coefficients

23

Figure 10: The lift coefficient of solutions computed with the 3rd-order scheme on Grids A-D. As a reference we show the Euler solution on Grid A. 


\section{Conclusions}

Finite difference methods satisfying the SBP property with boundary conditions enforced by the SAT procedure are used to solve the compressible Navier-Stokes and Euler equations on multi-block grids. To validate the code, we ran the benchmark case, $M a=0.5, R e=5000$ around a NACA0012, and showed that it was accurately computed. Next, we moved on to study the case $M a=0.5$ and $R e=10000$.

We studied the accuracy of solutions on several different grids and computed with the 3rd- or the 5th-order schemes. We measured the separation point as well as the effective amount of artificial diffusion. The accuracy of those quantities correlated well with the resolution of the boundary layer. By that we conclude that by evaluating the enforcement of the no-slip condition one can get a good indication of the accuracy of a computation. This is very useful in practice since an expensive grid refinement study is not required to assess the accuracy of the scheme.

The Euler equations have earlier been shown to be a good model for vortex propagation [3], but when it interacts with the airfoil the viscous effects become important. We computed the lift and drag coefficients as the vortex passed the airfoil and although on a large scale the Euler and

Navier-Stokes equations suggest roughly the same behavior, the viscosity of the Navier-Stokes equations adds small scale structures to the flow.

\section{References}

[1] M. H. Carpenter, J. Nordström, and D. Gottlieb. A Stable and Conservative Interface Treatment of Arbitrary Spatial Accuracy. J. Comput. Phys., 148, 1999.

[2] G. Erlebacher, M. Y. Hussaini, and C.-W. Shu. Interaction of a shock with a longitudinal vortex. J. Fluid. Mech., 337:129-153, 1997.

[3] K. Mattsson, M. Svärd, M.H. Carpenter, and J. Nordström. High-order accurate computations for unsteady aerodynamics. Computers and Flu$i d s, 36(3): 636-649,2007$.

[4] K. Mattsson, M. Svärd, and J. Nordström. Stable and Accurate Artificial Dissipation. Journal of Scientific Computing, 21(1):57-79, August 2004. 
[5] S. Mishra and M. Svärd. On stability of numerical schemes via frozen coefficients and the magnetic induction equations. under review Mathematics of Computations, 2008.

[6] S. Mittal. Finite element computation of unsteady viscous compressible flows. Comput. Methods Appl. Engrg., 157:151-175, 1998.

[7] J. Nordström and M. H. Carpenter. High-order finite difference methods, multidimensional linear problems, and curvilinear coordinates. $J$. Comput. Phys., 173, 2001.

[8] G. Strang. Accurate partial difference methods II. non-linear problems. Num. Math., 6:37-46, 1964.

[9] M. Svärd, M.H. Carpenter, and J. Nordström. A stable high-order finite difference scheme for the compressible Navier-Stokes equations, far-field boundary conditions. Journal of Computational Physics, 225:1020-1038, 2007.

[10] M. Svärd and J. Nordström. On the order of accuracy for difference approximations of initial-boundary value problems. J. Comput. Physics, 218(1):333-352, October 2006.

[11] M. Svärd and J. Nordström. A stable high-order finite difference scheme for the compressible Navier-Stokes equations, wall boundary conditions. J. Comput. Phys., 227:4805-4824, 2008.

[12] R. C. Swanson and E. Turkel. A multistage time-stepping scheme for the Navier-stokes equations. In AIAA 23rd Aerospace sciences meeting, Reno, Nevada, USA, January 1985. AIAA.

[13] R. C. Swanson and E. Turkel. Artificial dissipation and central difference schemes for the Euler and Navier-stokes equations. In Computational Fluid Dynamics Conference, 8th, Honolulu, USA, June 1987. AIAA.

[14] L. Tang and J.D. Baeder. Adaptive Euler simulations of airfoil-vortex interaction. International Journal for Numerical Methods in Fluids, 53:777-792, 2007.

[15] V. Venkatakrishnan. Viscous computations using a direct solver. Comput. Fluids, 18(2):191-204, 1990. 